\title{
Locoregional recurrence-associated factors and risk-adapted postmastectomy radiotherapy for breast cancer staged in CTI-2N0-I after neoadjuvant chemotherapy
}

This article was published in the following Dove Press journal:

Cancer Management and Research

\section{Xin Wang \\ Liming $X u$ \\ Zhenzhen Yin \\ Daquan Wang \\ Qi Wang \\ Kunpeng $\mathrm{Xu}$ \\ Jinlin Zhao, \\ Lujun Zhao \\ Zhiyong Yuan \\ Ping Wang}

Department of Radiation Oncology, Tianjin Medical University Cancer Institute and Hospital, National Clinical Research Center for Cancer, Key Laboratory of Cancer Prevention and Therapy, Tianjin, Tianjin's Clinical Research Center for Cancer, Key Laboratory of Breast Cancer, Prevention and Therapy,

Tianjin Medical University, Ministry of Education, Tianjin, 300060, China
Correspondence: Ping Wang Department of Radiation Oncology, Tianjin Medical University Cancer Institute and Hospital, National Clinical Research Center for Cancer, Key Laboratory of Cancer Prevention and Therapy, Tianjin, Tianjin's Clinical Research Center for Cancer, Key Laboratory of Breast Cancer Prevention and Therapy, Tianjin Medical University, Ministry of Education, Huanhuxi Road,

Tianjin 300060, China

Tel $+86 \quad 1862222 \quad$ III 2

Fax +8622 23345607

Email wangping@tjmuch.com
Objective: In order to identify risk factors associated with locoregional recurrence (LRR) and assess the role of postmastectomy radiotherapy (PMRT) in early breast cancer (BC), managed with neoadjuvant chemotherapy (NAC) and mastectomy, a retrospective analysis of BC diagnosed with clinical stage T1-2N0-1 was conducted.

Patients and methods: A total of 217 patients were included in this analysis. The median age was 50 years (24-72 years). The clinical stage distributions were cT1 in 15 cases, cT2 in 202, cN0 in 53, and cN1 in 161 cases. All patients were treated with NAC and mastectomy, and 128 patients received PMRT.

Results: With a median follow-up time of 61 months, the 5-year cumulative LRR rate was $12 \%$. Multivariate analysis demonstrated that pathological N stage, lymph-vascular invasion, and histological grade were independent prognostic factors associated with LRR. A nomogram model based on these factors was established, based on which the patients were deeply stratified into low- and high-risk group. In the low-risk group, radiotherapy did not decrease LRR (3.3\% in PMRT group, $1.7 \%$ in no PMRT group, $P=0.192$ ). While in the high-risk group, PMRT significantly decreased LRR (21.8\% in PMRT group, $42.2 \%$ in no PMRT group, $P=0.031)$.

Conclusion: Lymph-vascular invasion, histological grade, as well as pathological $\mathrm{N}$ stage were important prognostic factors associated with LRR in BC patients staged in cT1-2N0-1, who were managed with NAC and mastectomy. In our cohort, not only clinical and pathological stage information but also other risk factors were taken into consideration when adjuvant PMRT was recommended. In the high-risk subgroup, PMRT significantly improved the prognosis.

Keywords: breast cancer, neoadjuvant chemotherapy, postmastectomy radiotherapy, prognosis

\section{Introduction}

In recent decades, neoadjuvant chemotherapy (NAC) has become common for treatment of breast cancer (BC). With the downstage of NAC, some inoperable diseases may regain chances of surgery, and those who would have originally required mastectomy maybe able to undergo breast-conserving surgery (BCS). ${ }^{1-5}$ Therefore, NAC has been used for locally advanced diseases and also early-staged BC. ${ }^{6,7}$ However, there were also some concerns of NAC such as cancer may progress, potential of over- or under-treatment, and limited evidence base to guide adjuvant treatment. Furthermore, upfront surgery followed by adjuvant chemotherapy assured an accurate assessment of disease at the time of initial treatment. Due to the inconsistency of clinical evaluation of the disease extent both at diagnosis and post-NAC, the evaluation of locoregional 
recurrence (LRR) risks becomes more complex. Though it is well established that patients with stage III/IV, or positive node $\geq 4$, harboring high LRR rates and postmastectomy radiotherapy (PMRT) show significantly reduced LRR and improved survival, ${ }^{8-12}$ there is little information available on stage I-II disease after NAC and mastectomy. For cT1-2N0-1 disease, even in the adjuvant settings, the value of PMRT has remained an issue of controversy until now. The addition of NAC in this subgroup will significantly mask the indication for PMRT and complicate the situation. LRR risks at the time of presentation and post-NAC, as well as biologic response to NAC, should be taken into consideration. This may lead to the recommendation of PMRT in early BC after NAC is determined on a case-by-case basis.

In order to evaluate the LRR rate and identify associated risk factors, a retrospective analysis of cT1-2N0-1 BC postNAC and mastectomy was conducted, helping to provide some evidence for the recommendation of adjuvant PMRT.

\section{Patients and methods}

\section{Patient characteristics}

Patients with BC staged in cT1-2N0-1M0 and treated with mastectomy after NAC in our institute between 2011 and 2013 were retrospectively analyzed. All patients underwent mammography and breast ultrasonography prior to chemotherapy. Clinical nodal status was determined by physical examination and ultrasound. Patients with distant metastasis, inflammatory or bilateral breast cancer, and previous or concurrent malignancy were excluded. A total of 217 patients met the inclusion criteria. The clinical stage was determined according to American Joint Committee on Cancer criteria (seventh edition). The clinical stage distributions were cT1 in 15 , cT2 in 202, cN0 in 53, and cN1 in 164 patients. This study was approved by Tianjin Medical University Cancer Institute and Hospital's Ethics Committee. And a waiver for individual patients' consent for this retrospective study was also obtained from this committee. To maintain confidentiality, relevant medical records, laboratory results, images, and histopathological data were collected anonymously. The records of patients were kept confidential, and individuals outside this research team had no access to them.

\section{Treatment details}

NAC regimes consisted of anthracycline and taxane chemotherapy ( $82 \%$ of cases) and cyclophosphamide, methotrexate, and fluorouracil (12\% of cases). All patients underwent mastectomy after NAC, with a median dissected lymph node number of 22. PMRT was determined by patient and her radiation oncologist; 128 (59\%) patients received PMRT and $89(41 \%)$ patients did not. Treatment volumes included the chest wall and regional nodal basins (high axilla and supraclavicular fossa, with or without the internal mammary chain). Dose prescription was 50 Gy in 25 fractions. Additionally, 213 cases (98\%) received adjuvant chemotherapy and the median number of chemotherapy (NAC + adjuvant chemotherapy) cycles was 6 . In cases of hormone receptor positive (estrogen and/or progesterone receptor positive), 122 cases $(73 \%)$ were treated with endocrine therapy.

\section{Statistical analysis}

LRR was defined as disease recurrence on the ipsilateral chest wall or ipsilateral axillary, supraclavicular, infraclavicular, or internal mammary lymph nodes. Time to follow-up was calculated from the date of diagnosis. The LRR rates were calculated using the Kaplan-Meier method. Univariate and multivariate analyses were performed using the Cox proportional hazards model. Factors with $P$-value $<0.05$ were included in multivariate analysis. According to the final set of the Cox regression model, a prognostic nomogram to predict risks of LRR was developed, and the accuracy of the prognostic model was evaluated using the concordance index (C-index). SPSS version 20.0 software and $\mathrm{R}$ version 3.3.2 were used for all statistical analyses.

\section{Results}

\section{Patient characteristics and treatment data}

Patient characteristics and treatment data are summarized in Table 1. When compared with patients who did not receive PMRT, a greater percentage of irradiated patients had more advanced clinical $\mathrm{N}$-stage $(P=0.004)$, clinical stage $(P=0.040)$, and ypN stage $(P=0.000)$. No difference between the two groups was observed in other clinical-pathological factors (Table 1).

\section{Follow-up and patterns of failures}

With a median follow-up of 61 months, the 5-year cumulative rate of locoregional recurrence-free survival (LRFS), distant metastasis-free survival, disease-free survival, and overall survival rates were $88 \%, 79 \%, 73 \%$, and $81 \%$, respectively. Twenty-six patients (12\%) developed LRR, and the most common site of LRR was chest wall (17 patients, 65\%), followed by the supraclavicular lymph node (nine patients, $35 \%$ ). Axillary nodal and inner mammary nodal failure occurred in three patients each. Eleven patients presented with isolated LRR, and other 15 patients also experienced distant metastases (Figure 1). 
Table I Patients' clinical-pathological characteristics and treatment data

\begin{tabular}{|c|c|c|c|c|c|c|}
\hline \multirow[t]{2}{*}{ Characteristics } & \multirow[t]{2}{*}{ Total } & \multicolumn{2}{|c|}{ No PMRT (89 patients) } & \multicolumn{2}{|c|}{ PMRT (I 28 patients) } & \multirow[t]{2}{*}{$P$-value } \\
\hline & & No. & (\%) & No. & (\%) & \\
\hline Age group (years) & & & & & & 0.218 \\
\hline$\leq 50$ & 111 & 41 & 46.1 & 70 & 54.7 & \\
\hline$>50$ & 106 & 48 & 53.9 & 58 & 45.3 & \\
\hline Menopausal status & & & & & & 0.213 \\
\hline Premenopause & 126 & 49 & 55.1 & 77 & 60.2 & \\
\hline Postmenopause & 89 & 38 & 42.7 & 51 & 39.8 & \\
\hline Unknown & 2 & 2 & 2.2 & 0 & 0.0 & \\
\hline Clinical T stage & & & & & & 1.000 \\
\hline TI & 15 & 6 & 6.7 & 9 & 7.0 & \\
\hline $\mathrm{T} 2$ & 202 & 83 & 93.3 & 119 & 93.0 & \\
\hline Clinical N stage & & & & & & 0.004 \\
\hline No & 53 & 31 & 34.8 & 22 & 17.2 & \\
\hline NI & 164 & 58 & 65.2 & 106 & 82.8 & \\
\hline Clinical stage & & & & & & 0.040 \\
\hline I & 5 & 4 & 4.5 & I & 0.8 & \\
\hline lla & 58 & 29 & 32.6 & 29 & 22.7 & \\
\hline Ilb & 154 & 56 & 62.9 & 98 & 76.6 & \\
\hline NAC cycles & & & & & & 0.214 \\
\hline$<4$ & 105 & 48 & 53.9 & 57 & 44.5 & \\
\hline$\geq 4$ & 112 & $4 I$ & 46.1 & 71 & 55.5 & \\
\hline урТ stage & & & & & & 0.069 \\
\hline урТ0-2 & 200 & 86 & 96.6 & 114 & 89.1 & \\
\hline урТ3-4 & 17 & 3 & 3.4 & 14 & 10.9 & \\
\hline ypN stage & & & & & & 0.000 \\
\hline ypNo & 58 & 42 & 47.2 & 16 & 12.5 & \\
\hline ypNI & 61 & 38 & 42.7 & 23 & 18.0 & \\
\hline ypN2-N3 & 98 & 9 & 10.1 & 89 & 69.5 & \\
\hline Histological grade & & & & & & 0.257 \\
\hline I & 2 & 2 & 2.2 & 0 & 0.0 & \\
\hline 2 & 178 & 69 & 77.5 & 109 & 85.2 & \\
\hline 3 & 17 & 8 & 9.0 & 9 & 7.0 & \\
\hline Unknown & 20 & 10 & 11.2 & 10 & 7.8 & \\
\hline LVI & & & & & & 0.252 \\
\hline Yes & 21 & 6 & 6.7 & 15 & 11.7 & \\
\hline No & 196 & 83 & 83.3 & 113 & 88.3 & \\
\hline ER status & & & & & & 0.923 \\
\hline Positive & 167 & 68 & 76.4 & 99 & 77.3 & \\
\hline Negative & 49 & 21 & 23.6 & 28 & 21.9 & \\
\hline Unknown & 1 & 0 & 0.0 & 1 & 0.8 & \\
\hline PR status & & & & & & 0.790 \\
\hline Positive & 157 & 63 & 70.8 & 94 & 73.4 & \\
\hline Negative & 59 & 26 & 29.2 & 33 & 25.8 & \\
\hline Unknown & 1 & 0 & 0.0 & 1 & 0.8 & \\
\hline Her- 2 receptor status & & & & & & 0.616 \\
\hline Positive & 23 & 11 & 12.4 & 12 & 9.4 & \\
\hline Negative & 142 & 60 & 67.4 & 82 & 64.1 & \\
\hline $\operatorname{IHC}(2+) \mathrm{a}$ & 47 & 17 & 19.1 & 30 & 23.4 & \\
\hline Unknown & 5 & 1 & 1.1 & 4 & 3.1 & \\
\hline Cycles of chemotherapy & & & & & & 0.139 \\
\hline$<6$ & 36 & 19 & 21.3 & 17 & 13.3 & \\
\hline$\geq 6$ & $|8|$ & 70 & 78.7 & 111 & 86.7 & \\
\hline Hormonal therapy & & & & & & 0.851 \\
\hline Yes & 122 & 48 & 53.9 & 74 & 57.8 & \\
\hline No & 90 & 39 & 43.8 & 51 & 39.8 & \\
\hline Unknown & 5 & 2 & 2.2 & 3 & 2.3 & \\
\hline Targeted therapy & & & & & & 0.810 \\
\hline Yes & 12 & 6 & 6.7 & 6 & 4.7 & \\
\hline No & 202 & 82 & 92.1 & 120 & 93.8 & \\
\hline Unknown & 3 & 1 & 1.1 & 2 & 1.6 & \\
\hline
\end{tabular}

Abbreviations: PMRT, postmastectomy radiotherapy; NAC, neoadjuvant chemotherapy; LVI, lymph-vascular invasion; ER, estrogen receptor; PR, progesterone receptor. 


\section{Univariable and multivariable analyses}

All clinical-pathological factors were included in univariate analysis (Table 2). Factors with $P$-value $<0.05$ in univariate analysis were then included in multivariate analysis. Pathological stage $(P=0.004)$, histological grade $(P=0.001)$, and lymph-vascular invasion (LVI) $(P=0.044)$ were identified as

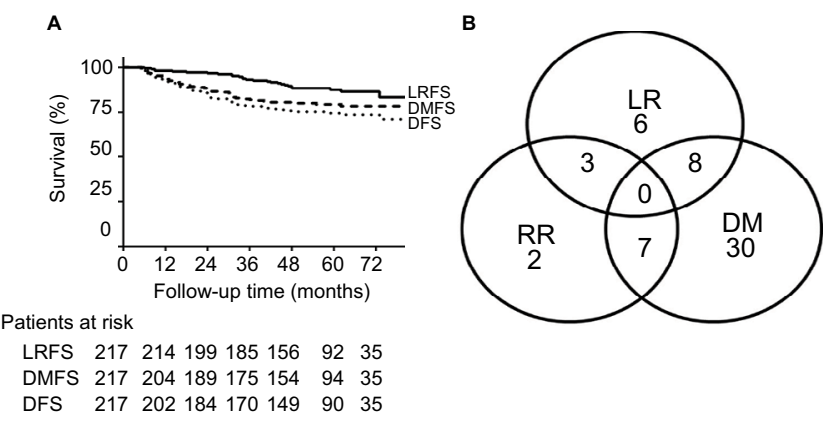

Figure I (A) Survival curves of the whole cohort of patients; (B) failure pattern of the whole cohort of patients.

Abbreviation: LRFS, locoregional recurrence-free survival; DMFS, distant metastasis free survival; DFS, disease free survival; LR, local recurrence; RR, regional recurrence; DM, distant metastasis. independent prognosis factors associated with LRR (Table 3). The LRR curves were plotted by Kaplan-Meier method (Figure 2). The 5y-LRR rates were significantly different according to pathological $\mathrm{N}$ stage (ypN0, ypN1, and ypN2-3: $2 \%$, $5 \%$, and $25 \%$, respectively, $P=0.000$ ), LVI (yes and no: $33 \%$ and $11 \%$, respectively, $P=0.001$ ), and histological grade (grade 3 and non-grade $3: 42 \%$ and $11 \%$, respectively, $P=0.000$ ).

\section{Nomogram model for predicting LRR risk and risk-adapted PMRT}

Based on the prognostic factors, a nomogram predicting locoregional failure for early staged $\mathrm{BC}$ after NAC and mastectomy was developed (Figure 3A). The C-index was 0.784 . Risk scores were calculated for each patient and a cutoff value of 80 was selected according to ROC curve. Patients were deeply stratified into low risk (risk scores $<80,48 \%$ ) and high-risk subgroups (risk scores $>80,52 \%$ ) according to the nomogram. Low-risk patients had significantly lower LRR rate than high-risk patients ( $5 \mathrm{y}-\mathrm{LRR}: 3 \%$ vs $27 \%, P=0.000$ ).

Table 2 Univariate analysis of factors associated with LRFS

\begin{tabular}{|c|c|c|c|c|c|c|c|}
\hline Variables & $\begin{array}{l}\text { No. of } \\
\text { patients }\end{array}$ & $\begin{array}{l}\text { 5-Year } \\
\text { LRFS rate }\end{array}$ & $P$-value & Variables & $\begin{array}{l}\text { No. of } \\
\text { patients }\end{array}$ & $\begin{array}{l}\text { 5-Year } \\
\text { LRFS rate }\end{array}$ & $P$-value \\
\hline Age group & & & 0.232 & LVI & & & 0.001 \\
\hline$\leq 50$ & 111 & 90.7 & & Yes & 21 & 67.3 & \\
\hline$>50$ & 106 & 83.8 & & No & 196 & 90.5 & \\
\hline Menopausal status & & & 0.139 & ER status & & & 0.190 \\
\hline Premenopause & 126 & 92.3 & & Positive & 167 & 90.3 & \\
\hline Postmenopause & 89 & 82.6 & & Negative & 49 & 81.4 & \\
\hline Clinical T stage & & & 0.488 & PR status & & & 0.530 \\
\hline TI & 15 & 93.3 & & Positive & 157 & 89.7 & \\
\hline $\mathrm{T} 2$ & 202 & 89.2 & & Negative & 59 & 84.8 & \\
\hline Clinical $\mathrm{N}$ stage & & & 0.329 & Her-2 recep & & & 0.160 \\
\hline No & 53 & 84.5 & & Positive & 23 & 68.9 & \\
\hline NI & 164 & 87.1 & & Negative & 142 & 88.6 & \\
\hline Clinical stage & & & 0.238 & Adjuvant ra & & & 0.181 \\
\hline 1 & 5 & 80.0 & & Yes & 128 & 86.7 & \\
\hline Ila & 58 & 86.7 & & No & 89 & 90.7 & \\
\hline Ilb & 154 & 86.2 & & Adjuvant ch & & & 0.589 \\
\hline NAC cycles & & & 0.579 & Yes & 213 & 89.4 & \\
\hline$<4$ & 105 & 86.8 & & No & 4 & 100.0 & \\
\hline$\geq 4$ & 112 & 88.0 & & Cycles of ch & & & 0.272 \\
\hline урТ stage & & & 0.000 & $<6$ & 36 & 93.8 & \\
\hline урТ0-2 & 200 & 90.1 & & $\geq 6$ & 181 & 87.5 & \\
\hline урТ3-4 & 17 & 56.0 & & Hormonal $\mathrm{t}$ & & & 0.120 \\
\hline ypN stage & & & 0.000 & Yes & 122 & 90.4 & \\
\hline ypNo & 58 & 98.3 & & No & 90 & 82.2 & \\
\hline ypNI & 61 & 94.7 & & Targeted th & & & 0.152 \\
\hline ypN2-3 & 98 & 75.0 & & Yes & 12 & 71.3 & \\
\hline Histological grade & & & 0.000 & No & 202 & 89.2 & \\
\hline $\mathrm{I}+2$ & 180 & 89.6 & & & & & \\
\hline 3 & 17 & 58.2 & & & & & \\
\hline
\end{tabular}

Abbreviations: NAC, neoadjuvant chemotherapy; LVI, lymph-vascular invasion; LRFS, locoregional recurrence-free survival; ER, estrogen receptor; PR, progesterone receptor. 
Additionally, we evaluated the benefits of PMRT in different risk groups. In the low-risk group, PMRT did not influence 5y-LRR (3.3\% in PMRT group, $1.7 \%$ in no PMRT group, $P=0.192)$. In the high-risk group, PMRT significantly decreased 5y-LRR (21.8\% in PMRT group vs $42.2 \%$ in no PMRT group, $P=0.031$ ) (Figure 3B). Similar benefit trends were also found in overall survival; PMRT significantly decreased deaths in high-risk group, but not in low-risk group (Figure S1).

Table 3 Multivariate analysis of LRFS

\begin{tabular}{llll}
\hline Variable & HR & $\mathbf{9 5 \%} \mathbf{C l}$ & $\boldsymbol{P}$-value \\
\hline yPN & & & 0.004 \\
ypN stage NI vs N0 & 3.602 & $0.40 \mathrm{I}-32.309$ & 0.252 \\
ypN stage N2-3 vs N0 & 13.730 & $\mathrm{I} .832-102.903$ & $0.01 \mathrm{I}$ \\
Histological grade 3 vs I-2 & 4.598 & $\mathrm{I} .808-1 \mathrm{I} .693$ & $0.00 \mathrm{I}$ \\
LVI yes vs no & 2.654 & $\mathrm{I} .029-6.847$ & 0.044 \\
\hline
\end{tabular}

Abbreviations: LRFS, locoregional recurrence-free survival; LVI, lymph-vascular invasion.

\section{Discussion}

To our knowledge, this retrospective study, including a highly selective subgroup of $\mathrm{BC}$, presents the largest single cohort of stage cT1-2N0-1 cases after NAC and mastectomy. NAC is increasingly used in early $\mathrm{BC}$, making more patients eligible for BCS. For cases with poor response to chemotherapy, mastectomy is still the main treatment of choice. ${ }^{3}$ However, the LRR rate and value of PMRT in early-stage BC after $\mathrm{NAC}$ and mastectomy remain contentious. In our cohort, the 5 -year LRR rate was $12 \%$. ypN stage, histological grade, and LVI were identified as independent prognostic factors associated with LRR. A nomogram model for predicting LRR was established, and patients were divided into low- and high-risk groups. PMRT significantly reduced LRR rates in the highrisk group, but not in the low-risk group.

Until now, early staged BC after NAC had been poorly studied. In the adjuvant settings, the LRR rates ranged from $3 \%$ to $20 \%$ in patients with $\mathrm{T} 1-2$ with $1-3$ positive axillary
A

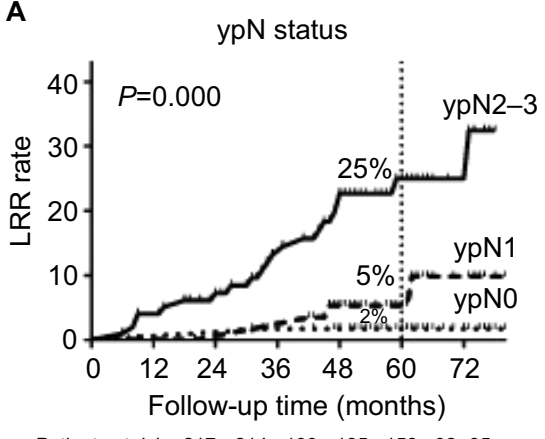

Patients at risk $217 \quad 214 \quad 199 \quad 185 \quad 158 \quad 92 \quad 35$
B

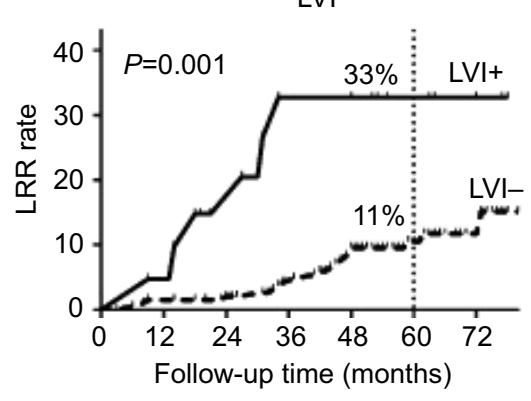

Patients at risk $217 \quad 214 \quad 199 \quad 185 \quad 158 \quad 92 \quad 35$
C

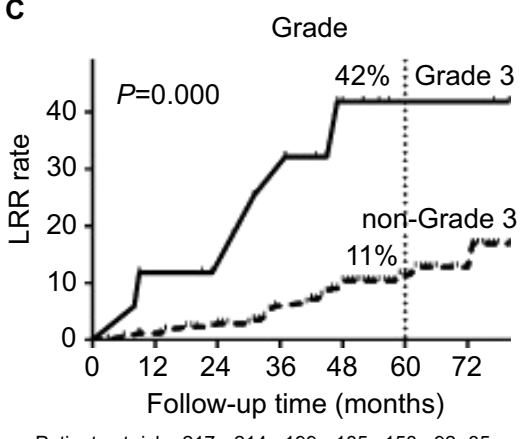

Figure 2 (A) LRR rates of patients in ypN0, ypNI, and ypN2-3; (B) LRR rates of patients with or without LVI; (C) LRR rates of patients with or without histological grade 3. Abbreviations: LRR, locoregional recurrence; LVI, lymph-vascular invasion.

A

Points

ypN stage

ypN stage
Histologic grade

LVI

Total points

3y-LRFS

5y-LRFS
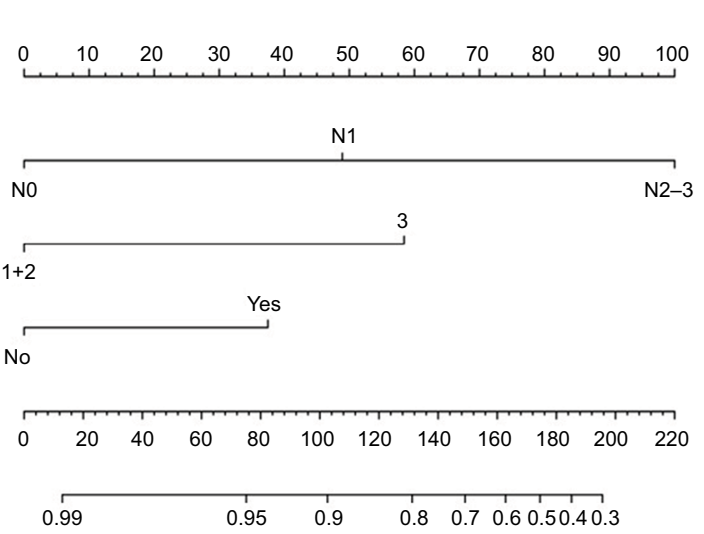

$\begin{array}{llllllllllll}0.95 & 0.9 & 0.8 & 0.7 & 0.6 & 0.5 & 0.4 & 0.3 & 0.2 & 0.10 .05\end{array}$
B

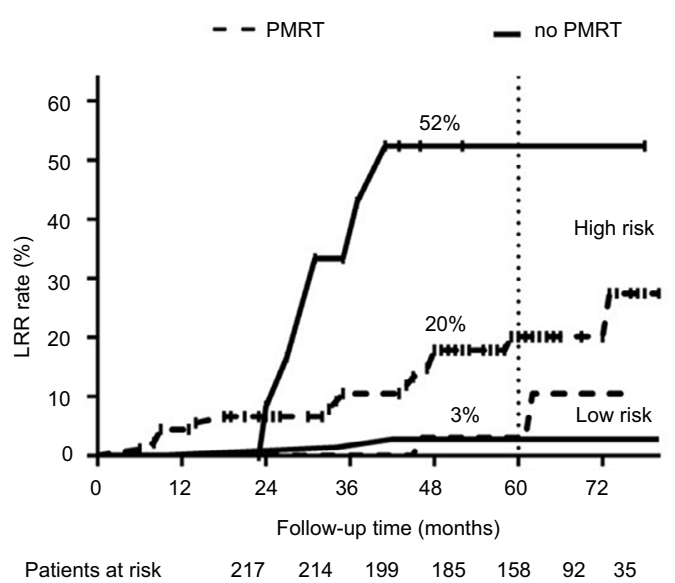

Figure 3 (A) A nomogram model was established according to our dataset. (B) The LRR curves in low- and high-risk group treated with or without PMRT. Abbreviations: LRR, locoregional recurrence; PMRT, postmastectomy radiotherapy, LRFS, locoregional recurrence-free survival. 
lymph nodes (ALN). ${ }^{13,14}$ Various risk factors such as age, grade, LVI, numbers of positive lymph node, and so on affect LRR. Similar results were also observed in NAC settings from retrospective analyses. Variables, including initial clinical stage, age, extent of residual disease, and risk factors (LVI, extra-capsular extension [ECE], and triple-negative phenotype), impacting LRR after NAC have been reported. Fowble et a ${ }^{15}$ suggested that in clinical stage II disease, age, estrogen receptor status, chemotherapy response, LVI, and ECE affect LRR after NAC. Another study focused on stage I-II disease identified clinical stage T3N0, $\geq 4$ positive lymph nodes after NAC, and young age as poor predictors for LRR. ${ }^{16}$ In another series of stage II patients, young age, LVI, and high grade were associated with an increased risk of LRR. ${ }^{11,17}$ Our analysis found similar results: pathological N stage, LVI, and tumor grade were independent factors influencing LRR in cT1-2N0-1 patients after NAC and mastectomy.

In order to establish a valid method to predict LRR risk, a nomogram model was developed based on risk factors. Several nomogram models have been proposed to predict the response of ALN, pCR, and DFS. ${ }^{18-20}$ In the present study, we presented the first nomogram model to predict LRR in clinical stage I-II patients after NAC and mastectomy, showing a high degree of accuracy with a $\mathrm{C}$-index of 0.78 . Patients with risk scores $<80$, namely, patients with none or only one of the three risk factors (ypN1, LVI, or grade 3) were categorized as low-risk group that disclosed LRR rate of 3\%. This is in accordance with a report, ${ }^{15}$ which suggested that patients in cT1-2N0-1 with pathologically N0, or 1-3 positive nodes and with ER+ disease, aged $>40$ years with no LVI or ECE presented the lowest risk category, with LRR rate $<10 \%$. Similar results were observed from Vila, for clinical stage II patients with ypN0 or ypN1: the 5y-LRR rate was $5 \% .{ }^{21}$ In contrast, patients with a risk score $>80$ were grouped as high risk with LRR rates emerging at $27 \%$. Our nomogram model was highly efficient in discriminating different LRR risks in cT1-2N0-1 after NAC and mastectomy.

Next, we attempted to make sense of recommendation of PMRT in early BC after NAC as a risk-adapted therapeutic strategy in the context of existing clinical knowledge. LRR plays a vital role in determining whether PMRT should be considered or not. It is generally accepted that risks $<10 \%$ do not warrant PMRT, whereas $>20 \%$ do. Unfortunately, due to limited information with relatively small sample size, controversies arise about the benefits of PMRT in BC staged I-II after NAC and mastectomy. In clinical practice, PRMT was determined on a case-by-case basis by patient's radiation oncologist, usually based on the maximum stage from the pre-therapy clinical and pathological stage. According to our model, $5 \mathrm{y}-\mathrm{LRR}$ rate reached at $27 \%$ in high-risk group and PMRT significantly decreased 5y-LRR rates from $42 \%$ to $22 \%$. Huang et $\mathrm{al}^{9}$ also demonstrated that PMRT significantly lowered LRR rates for patients with stage IIb, or with four or more positive nodes, which was consistent with high-risk patients in our cohort. In our analysis, lowrisk patients presented 3\% of LRR and showed no benefit from PMRT. Le Scodan et $\mathrm{a}^{22}$ analyzed patients with clinical stage II (63\%)-III (27\%) and ypN0 after NAC, observing no significant difference in LRFS for patients treated with or without PMRT (96.2\% vs $92.5 \%$ ). Shim et $\mathrm{a}^{23}$ also identified $151 \mathrm{BC}$ patients with clinical stage II (60\%)-III (40\%) and ypN0 disease: the 5-year LRFS rates were $98.1 \%$ with PMRT and $92.3 \%$ without PMRT. PMRT showed no correlation with LRFS. However, another report concluded that in cT1-2N1 patients, who received NAC and mastectomy, ${ }^{24}$ PMRT significantly improved LRFS ( $96.9 \%$ vs $78.6 \%$ ), even in ypN0 patients $(94.7 \%$ vs $72.9 \%)$. Therefore, prospective randomized trials are urgently warranted, and we look forward to the result of an ongoing Phase III clinical trial NSABP B-051/ RTOG 1304 (NCT01872975), which is focused on cT1-3N1 BC with N-negative after NAC treated with or without RT. This trial may provide strong clinical evidence to support decisions on PMRT after NAC in low-risk subgroup.

As a retrospective study, we acknowledge that our study has several limitations. First, bias exists between patients who received PMRT and those who did not. In addition, although NAC regimens are relatively standardized, chemotherapy cycles were inconsistent and might compromise chemotherapy response rates, and the follow-up time may not be long enough for BC. However, our study included relatively large sample sizes of cT1-2N0 BC after NAC and mastectomy and established a valid nomogram model to provide a risk-adapted recommendation of PMRT in this most disputable subgroup. We believe that the current analysis provides some evidence for risk-adapted PMRT and will be important in future validation from larger and prospective data sets.

\section{Conclusion}

In our cohort, LVI, histological grade, as well as pathological $\mathrm{N}$ stage were independently important prognostic factors associated with LRR in BC staged in cT1-2N0-1 after NAC and mastectomy. Risk-adapted PMRT is recommended according to our nomogram model, which could be a valuable tool for predicting risk of LRR and guiding optimal clinical decisions, and validations from other independent data sets are warranted. 


\section{Acknowledgments}

A part of this work was accepted for presentation in the poster viewing session in ESTRO 37th Annual Meeting in Spain.

This study is supported by the National Natural Science Foundation of China (Grant No. 81502656).

\section{Disclosure}

The authors report no conflicts of interest in this work.

\section{References}

1. van der Hage JA, Mieog JS, van de Vijver MJ, van de Velde CJ; European Organization for Research and Treatment of Cancer. Efficacy of adjuvant chemotherapy according to hormone receptor status in young patients with breast cancer: a pooled analysis. Breast Cancer Res. 2007;9(5):R70.

2. Golshan M, Cirrincione CT, Sikov WM, et al. Impact of neoadjuvant therapy on eligibility for and frequency of breast conservation in stage II-III HER2-positive breast cancer: surgical results of CALGB 40601 (Alliance). Breast Cancer Res Treat. 2016;160(2):297-304.

3. Rastogi P, Anderson SJ, Bear HD, et al. Preoperative chemotherapy: updates of National Surgical Adjuvant Breast and Bowel Project Protocols B-18 and B-27. J Clin Oncol. 2008;26(5):778-785.

4. Hayes DF, Schott AF. Neoadjuvant chemotherapy: what are the benefits for the patient and for the investigator? J Natl Cancer Inst Monogr. 2015;2015(51):36-39.

5. Kümmel S, Holtschmidt J, Loibl S. Surgical treatment of primary breast cancer in the neoadjuvant setting. Br J Surg. 2014;101(8):912-924.

6. Bear HD, Anderson S, Smith RE, et al. Sequential preoperative or postoperative docetaxel added to preoperative doxorubicin plus cyclophosphamide for operable breast cancer: National Surgical Adjuvant Breast and Bowel Project Protocol B-27. J Clin Oncol. 2006;24(13):2019-2027.

7. Gralow JR, Burstein HJ, Wood W, et al. Preoperative therapy in invasive breast cancer: pathologic assessment and systemic therapy issues in operable disease. J Clin Oncol. 2008;26(5):814-819.

8. Clarke M, Collins R, Darby S, et al. Effects of radiotherapy and of differences in the extent of surgery for early breast cancer on local recurrence and 15-year survival: an overview of the randomised trials. Lancet. 2005;366(9503):2087-2106.

9. Huang EH, Tucker SL, Strom EA, et al. Postmastectomy radiation improves local-regional control and survival for selected patients with locally advanced breast cancer treated with neoadjuvant chemotherapy and mastectomy. J Clin Oncol. 2004;22(23):4691-4699.

10. Mcguire SE, Gonzalez-Angulo AM, Huang EH, et al. Postmastectomy radiation improves the outcome of patients with locally advanced breast cancer who achieve a pathologic complete response to neoadjuvant chemotherapy. Int J Radiat Oncol Biol Phys. 2007;68(4):1004-1009.
11. Nagar H, Mittendorf EA, Strom EA, et al. Local-regional recurrence with and without radiation therapy after neoadjuvant chemotherapy and mastectomy for clinically staged T3N0 breast cancer. Int J Radiat Oncol Biol Phys. 2011;81(3):782-787.

12. Francis SR, Frandsen J, Kokeny KE, Gaffney DK, Poppe MM. Outcomes and utilization of postmastectomy radiotherapy for T3N0 breast cancers. Breast. 2017;32:156-161.

13. Trovo M, Durofil E, Polesel J, et al. Locoregional failure in early-stage breast cancer patients treated with radical mastectomy and adjuvant systemic therapy: which patients benefit from postmastectomy irradiation? Int J Radiat Oncol Biol Phys. 2012;83(2):e153-e157.

14. Hamamoto Y, Ohsumi S, Aogi K, et al. Are there high-risk subgroups for isolated locoregional failure in patients who had T1/2 breast cancer with one to three positive lymph nodes and received mastectomy without radiotherapy? Breast Cancer. 2014;21(2):177-182.

15. Fowble BL, Einck JP, Kim DN, et al. Role of postmastectomy radiation after neoadjuvant chemotherapy in stage II-III breast cancer. Int J Radiat Oncol Biol Phys. 2012;83(2):494-503.

16. Garg AK, Strom EA, McNeese MD, et al. T3 disease at presentation or pathologic involvement of four or more lymph nodes predict for locoregional recurrence in stage II breast cancer treated with neoadjuvant chemotherapy and mastectomy without radiotherapy. Int J Radiat Oncol Biol Phys. 2004;59(1):138-145.

17. Garg AK, Oh JL, Oswald MJ, et al. Effect of postmastectomy radiotherapy in patients $<35$ years old with stage II-III breast cancer treated with doxorubicin-based neoadjuvant chemotherapy and mastectomy. Int J Radiat Oncol Biol Phys. 2007;69(5):1478-1483.

18. Hennessy BT, Hortobagyi GN, Rouzier R, et al. Outcome after pathologic complete eradication of cytologically proven breast cancer axillary node metastases following primary chemotherapy. J Clin Oncol. 2005;23(36):9304-9311.

19. Rouzier R, Pusztai L, Delaloge S, et al. Nomograms to predict pathologic complete response and metastasis-free survival after preoperative chemotherapy for breast cancer. J Clin Oncol. 2005;23(33):8331-8339.

20. Jeruss JS, Mittendorf EA, Tucker SL, et al. Combined use of clinical and pathologic staging variables to define outcomes for breast cancer patients treated with neoadjuvant therapy. J Clin Oncol. 2008;26(2):246-252.

21. Vila J, Teshome M, Tucker SL, et al. Combining clinical and pathologic staging variables has prognostic value in predicting local-regional recurrence following neoadjuvant chemotherapy for breast cancer. Ann Surg. 2017;265(3):574-580.

22. Le Scodan R, Selz J, Stevens D, et al. Radiotherapy for stage II and stage III breast cancer patients with negative lymph nodes after preoperative chemotherapy and mastectomy. Int J Radiat Oncol Biol Phys. 2012;82(1):e1-e7.

23. Shim SJ, Park W, Huh SJ, et al. The role of postmastectomy radiation therapy after neoadjuvant chemotherapy in clinical stage II-III breast cancer patients with pN0: a multicenter, retrospective study (KROG 12-05). Int J Radiat Oncol Biol Phys. 2014;88(1):65-72.

24. Cao L, Ou D, Shen KW, et al. Outcome of postmastectomy radiotherapy after primary systemic treatment in patients with clinical T1-2N1 breast cancer. Cancer Radiother. 2018;22(1):38-44. 


\section{Supplementary material}

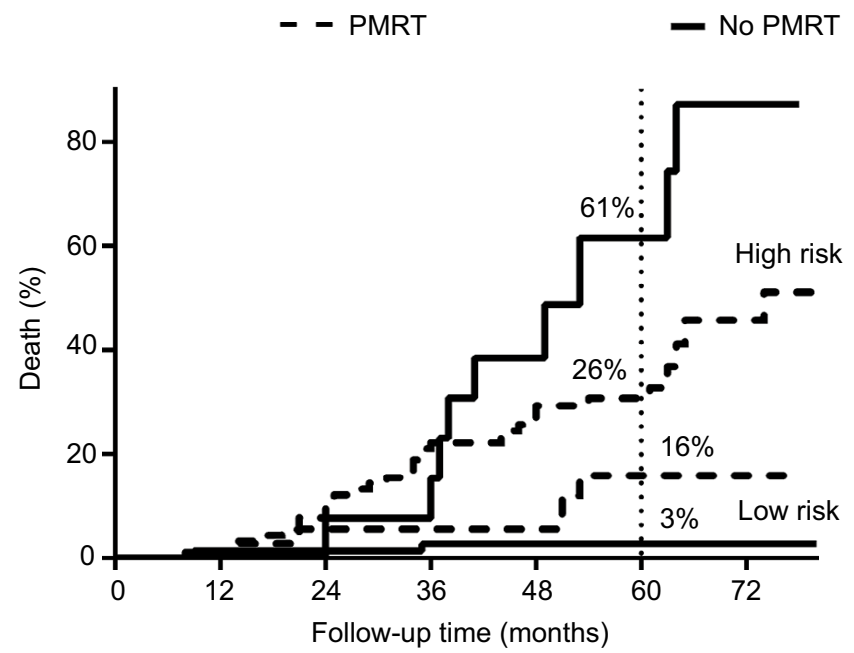

Patients at risk $21721419918515892 \quad 35$

Figure SI Mortality curves in low-risk and high-risk groups.

Notes: PMRT decreased mortality in high-risk group, but not in low-risk group.

Abbreviation: PMRT, postmastectomy radiotherapy.

\section{Publish your work in this journal}

Cancer Management and Research is an international, peer-reviewed open access journal focusing on cancer research and the optimal use of preventative and integrated treatment interventions to achieve improved outcomes, enhanced survival and quality of life for the cancer patient. The manuscript management system is completely online and includes

a very quick and fair peer-review system, which is all easy to use. Visit $\mathrm{http}: / / \mathrm{www}$.dovepress.com/testimonials.php to read real quotes from published authors. 